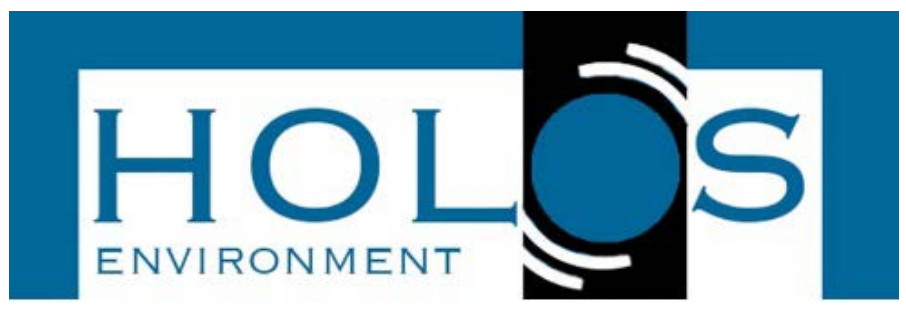

\title{
ANÁLISE DA SUSTENTABILIDADE HIDROAMBIENTAL DOS MUNICÍPIOS COM SEDE TOTALMENTE CONTIDA NA BACIA HIDROGRÁFICA DO RIO MOGI GUAÇU, SP
}

\section{ANALYSIS OF HYDRO-ENVIRONMENTAL SUSTAINABILITY OF MUNICIPALITIES WITH A TOTALLY HEADED HYDROGRAPHIC BASIN OF MOGI GUAÇU RIVER, SP}

\author{
Aloisio Calsoni Bozzini ${ }^{1}$, Fernanda Carla de Oliveira Prado ${ }^{2}$, João Paulo Pereira ${ }^{3}$, \\ Menoti Borri ${ }^{4}$, Cristina Filomena Pereira Rosa Paschoalato ${ }^{5}$
}

Artigo recebido em: 16/02/2018 e aceito para publicação em: 16/05/2018.

DOI: http://dx.doi.org/10.14295/holos.v18i1.12256

Resumo: Os gestores municipais e os cidadãos, a partir de suas instituições representativas, devem participar do Comitê de Bacia Hidrográfica da sua região, e, nesse sentido, têm o dever de promover melhorias na qualidade de vida e o equilíbrio ecológico. Alguns indicadores, agrupados nas dimensões/categorias desenvolvimento humano; econômico; abastecimento humano; pressões da irrigação, pecuária, abastecimento rural, aquicultura e lazer; coleta de esgoto; atendimento de coleta de lixo; e estado qualitativo da água, podem permitir uma análise e contribuir para a gestão dos recursos hídricos na bacia hidrográfica. O objetivo deste trabalho foi analisar a sustentabilidade hidroambiental dos municípios com sede totalmente contida na bacia hidrográfica do rio Mogi Guaçu, SP (Águas da Prata, Engenheiro Coelho, Luís Antônio, Mogi Mirim, Pitangueiras, São João da Boa Vista, Serra Negra, Sertãozinho, Socorro e Taquaral). A metodologia utilizada consistiu em uma pesquisa exploratória e documental, a partir do uso da estatística descritiva dentro de critérios e parâmetros de avaliação pré-definidos. Notou-se quanto ao Desenvolvimento Humano e os Indicadores Econômicos, uma situação preocupante na bacia, já que as médias dos municípios apresentaram performance de alerta. Em contrapartida, os indicadores de Saneamento apresentaram performance aceitável ou ideal. Finalmente, quanto aos indicadores referentes às Pressões da Irrigação, Pecuária, Abastecimento Rural, Aquicultura e Lazer a performance foi ideal na maioria dos municípios.

Palavras-chave: Sustentabilidade ambiental. Gestão Pública. Recursos hídricos.

Abstract: The municipal managers and the citizens, from their representative institutions, should participate

${ }^{1}$ UNIARARAS (Centro Universitário Hermínio Ometto) e UNIFEG (Centro Universitário da Fundação Educacional Guaxupé). Doutorando em Tecnologia Ambiental pela UNAERP (Universidade de Ribeirão Preto). Bolsista CAPES/PROSUP/UNAERP. E-mail: acbozzini@hotmail.com

${ }^{1}$ Instituto Federal de Educação, Ciência e Tecnologia de São Paulo (IFSP).Campus São João da Boa Vista. Doutoranda em Tecnologia Ambiental pela UNAERP (Universidade de Ribeirão Preto). E-mail: feroliveira2201@gmail.com

1 Instituto Federal de Educação, Ciência e Tecnologia de São Paulo (IFSP). Campus São João da Boa Vista. Doutorando em Tecnologia Ambiental pela UNAERP (Universidade de Ribeirão Preto). E-mail: administradorjoaopaulo@gmail.com

1 Instituto Federal de Educação, Ciência e Tecnologia de São Paulo (IFSP). Campus São João da Boa Vista. Doutorando em Tecnologia Ambiental pela UNAERP (Universidade de Ribeirão Preto). E-mail: menotib@gmail.com

1 Dra. da Pós-Graduação em Tecnologia Ambiental da UNAERP (Universidade de Ribeirão Preto). E-mail: cpaschoa@unaerp.br 
in the Hydrographic Basin Committee of their region, and, in this sense, they have the duty to promote improvements in the quality of life and the ecological balance. Some indicators, grouped in the dimensions / categories human development; economic; human supply; irrigation pressures, livestock, rural supply, aquaculture and leisure; sewage collection; garbage collection service; and qualitative status of water, can allow analysis and contribute to the management of water resources in the river basin. The objective of this work was to analyze the hydroenvironmental sustainability of the cities with a headquarter located in the Mogi Guaçu river basin, SP (Águas da Prata, Engenheiro Coelho, Luís Antônio, Mogi Mirim, Pitangueiras, São João da Boa Vista, Serra Negra, Sertãozinho , Socorro and Taquaral). The methodology used consisted of an exploratory and documentary research, based on the use of descriptive statistics within pre-defined criteria and evaluation parameters. Human Development and Economic Indicators were a worrying situation in the basin, since the municipal averages showed alert performance. On the other hand, the Sanitation indicators presented acceptable or ideal performance. Finally, with regard to the indicators related to the Pressures of Irrigation, Livestock, Rural Supply, Aquaculture and Leisure, the performance was ideal in most cities.

Keywords: Environmental sustainability. Public administration. Water resources.

\section{INTRODUÇÃO}

A água um dos recursos naturais mais intensamente utilizados pelo ser humano. É fundamental para a existência e a manutenção da vida, e para isso, deve estar presente no ambiente em quantidade e qualidade apropriadas.

Este recurso contribui para várias atividades, tais como: produção de energia, produção industrial, transporte fluvial, abastecimento de cidades (GARCIA et al., 2015).

Existem regiões no planeta com intensa demanda de água, tais como os grandes centros urbanos, os polos industriais a as zonas de irrigação. Essa demanda pode superar a oferta da água, sejam em termos quantitativos, seja porque a qualidade da água local está prejudicada em virtude da poluição. Tal degradação da sua qualidade pode afetar a oferta de água e também gerar graves problemas de desequilíbrio ambiental (poluição).

A Lei $n^{\circ}$ 9.433, de 8 de janeiro de 1997, institui a Política Nacional de Recursos Hídricos (PNRH) elencando seus objetivos, diretrizes gerais de ação e instrumentos para implementação, assim como as competências dos diversos poderes. Além disso, modifica as Leis 8.001/90 e 7.990/89 e regulamenta o inciso XIX do Art. 21 da Constituição Federal, a saber: "XIX - instituir sistema nacional de gerenciamento de recursos hídricos e definir critérios de outorga de direitos de seu uso".

Machado (2015) afirma que, existem consequências a respeito da conceituação da água como "bem de uso comum do povo", pois o uso da água não pode ser somente de uma pessoa, sendo ela física ou jurídica, o uso da água não pode ser significado de 
agressão, poluição, esgotamento entre outros, o uso da água deve ser motivada ou ter sua fundamentação dada pelo gestor público.

A Política Nacional de Recursos Hídricos se baseia nos fundamentos de que a água é um bem de domínio público limitado e dotado de valor econômico, que em situações de sua escassez é de uso primordial aos seres humanos e dos animais, devendo sempre ser seu uso múltiplo contando com a participação do poder público, de usuários e da comunidade que tem o dever de preservá-lo, tendo como unidade territorial para implementação da Política Nacional dos Recursos Hídricos e atuação do Sistema de Gerenciamento a bacia hidrográfica. Além disso, observa-se que os objetivos da PNRH focam o uso sustentável da água:

I - assegurar à atual e às futuras gerações a necessária disponibilidade de água, em padrões de qualidade adequados aos respectivos usos;

II - a utilização racional e integrada dos recursos hídricos, incluindo o transporte aquaviário, com vistas ao desenvolvimento sustentável;

III - a prevenção e a defesa contra eventos hidrológicos críticos de origem natural ou decorrentes do uso inadequado dos recursos naturais (BRASIL, 1997).

Neste sentido, cabe aos gestores municipais participação direta e permanente no Comitê de Bacia Hidrográfica para entendimento da realidade nesta unidade territorial a fim de promover o desenvolvimento sustentável, ou seja, utilizar os recursos naturais para geração de emprego/renda sem causar degradação ambiental para as presentes e futuras gerações. Para tanto, torna-se necessário aos gestores a apropriação de dados para uma análise do estado atual das questões da Bacia hidrográficas.

Segundo Carvalho et al. (2015), os indicadores distribuídos em sete dimensões/categorias (desenvolvimento humano; econômico; abastecimento humano; pressões da irrigação, pecuária, abastecimento rural, aquicultura e lazer; coleta de esgoto; atendimento de coleta de lixo; e estado qualitativo da água) podem permitir a inferência sobre as implicações diretas capazes de contribuirem para a gestão dos recursos hídricos na bacia hidrográfica.

Para Dowbor (2013), a utilização dos indicadores possibilita à contabilidade econômica ser um instrumento de cidadania, ao conceder às pessoas a oportunidade de avaliar, em termos de resultados finais para a sociedade, as iniciativas dos diversos atores públicos e privados. Segundo Souza et al (2014) a necessidade de desenvolver indicadores de sustentabilidade foi reconhecida pela Organização das Nações Unidas (ONU), no 
documento "Agenda 21", como resultado da Conferência das Nações Unidas sobre o Meio Ambiente e Desenvolvimento, realizado no Rio de Janeiro, em 1992.

Diante do exposto, o objetivo do artigo é analisar a sustentabilidade hidroambiental dos municípios com sede totalmente contida na bacia hidrográfica do rio Mogi Guaçu, SP.

\section{MATERIAL E MÉTODOS}

A metodologia utilizada neste estudo consistiu em uma pesquisa exploratória, e documental, a partir do uso da estatística descritiva dentro de critérios e parâmetros de avaliação pré-definidos e realizados por Carvalho et al. (2015) no trabalho realizado na subbacia do Alto Piranhas, no Estado da Paraíba. Foram selecionados alguns indicadores hidroambientais, de acordo com a realidade territorial e disponibilidade de dados (censos, institutos, agências, dentre outros), a fim de avaliar a sustentabilidade hidroambiental dos municípios com sede totalmente contida na bacia hidrográfica do Rio Mogi Guaçu, SP.

Os estudos que embasaram a escolha dos indicadores foram os trabalhos de Carvalho et al. (2015), Magalhães Júnior (2010), Vieira e Studart (2009), Guimarães (2008), Martins e Cândido (2008).

Para este trabalho, foram levantados dados cujas diferenças temporais fossem as mínimas possíveis. Esses dados variaram entre os anos de 2000 a 2017 . As fontes e os respectivos anos encontram-se no Quadro 1.

A definição e representação gráfica do índice de sustentabilidade hidroambiental foi adotada a metodologia proposta por Martins e Cândido (2008).

Observou-se que as variáveis estudadas apresentam diferentes unidades de medida, fazendo com que fosse necessária a utilização de unidades de agregação a partir das relações positivas e negativas de cada uma delas. A sequência adotada seguiu o entendimento de Sepúlveda (2005), Waquil et al. (2007) e Martins e Cândido (2008).

Segundo Carvalho et al. (2015) essa estratégia adotada proporciona o ajuste de valores observados das variáveis às escalas, cujo valor mínimo é zero e valor máximo é um. Ou seja, cria condições para a agregação nas dimensões/categorias, e, consequentemente, a estimação de um índice, que no caso do estudo foi denominado de Índice de Sustentabilidade Hidroambiental para Municípios (ISHM). 
De acordo com as propostas metodológicas apresentadas por Sepúlveda (2005), Waquil et al. (2007) e Martins e Cândido (2008), quando as variáveis são escolhidas, devese definir o tipo de relação que cada uma delas tem com a temática que se pretende investigar, no caso do estudo com a gestão da água. Nesse sentido, para cada variável é necessário identificar se ela mede uma situação em que, ao aumentar seu valor, favorece ou desfavorece o processo de desenvolvimento, no caso do estudo de sustentabilidade hidroambiental. Assim, existe uma relação positiva se um aumento no valor da variável resulta em melhoria do sistema; em contrapartida, a relação é negativa se um aumento no valor da variável resulta em piora do sistema. A operacionalização metodológica foi feita conforme Equações 1 e 2:

$$
\begin{aligned}
& \text { Relação Positiva: I = (x-m)/(M-m) } \\
& \text { Relação Negativa: I = (M-x)/(M-m) }
\end{aligned}
$$

Onde:

I = índice calculado referente a cada variável, para cada território analisado;

$\mathrm{x}$ = valor observado de cada variável em cada território analisado;

$\mathrm{m}=$ valor mínimo considerado;

$\mathrm{M}=$ valor máximo considerado.

Os indicadores escolhidos e as respectivas categorias/dimensões foram distribuídos de acordo com o Quadro1.

Quadro 1 - Indicadores do modelo de avaliação hidroambiental

\begin{tabular}{|c|c|c|c|}
\hline $\begin{array}{l}\text { CATEGORIAI } \\
\text { DIMENSÃO }\end{array}$ & INDICADOR & FONTE & $\begin{array}{c}\text { TIPO DE } \\
\text { RELAÇÃO DO } \\
\text { INDICADOR }\end{array}$ \\
\hline \multirow{4}{*}{$\begin{array}{c}\text { DESEMPENHO DO } \\
\text { SISTEMA QUANTO AO } \\
\text { DESENVOLVIMENTO } \\
\text { HUMANO }\end{array}$} & Taxa de Alfabetização & IBGE (2000) & Positiva \\
\hline & $\begin{array}{l}\text { Taxa de Mortalidade } \\
\text { Infantil (por mil) }\end{array}$ & DATASUS (2014) & Negativa \\
\hline & $\begin{array}{l}\text { IDH-M (Índice de } \\
\text { Desenvolvimento } \\
\text { Humano Municipal) }\end{array}$ & IBGE (2010) & Positiva \\
\hline & $\begin{array}{c}\text { Esperança de Vida ao } \\
\text { Nascer }\end{array}$ & $\begin{array}{c}\text { Atlas Brasil } \\
\text { (PNUD, IPEA E } \\
\text { FJP - 2010) }\end{array}$ & Positiva \\
\hline
\end{tabular}
(continua) 


\begin{tabular}{||c|c|c|c|}
\hline & $\begin{array}{c}\text { Quantidade de } \\
\text { estabelecimentos de } \\
\text { saúde (SUS) }\end{array}$ & IBGE (2009) & Positiva \\
\cline { 2 - 4 } & $\begin{array}{c}\text { Taxa de hospitalização } \\
\text { por desidratação em } \\
\text { menores de 5 anos }\end{array}$ & DATASUS (2000) & Negativa \\
\cline { 2 - 4 } & $\begin{array}{c}\text { Taxa de mortalidade } \\
\text { infantil por diarreia (por } \\
1.000 \text { nascidos vivos) }\end{array}$ & DATASUS (2000) & Negativa \\
\cline { 2 - 4 } & $\begin{array}{c}\text { População coberta pelo } \\
\text { Programa de Saúde da } \\
\text { Família (PSF) }\end{array}$ & DATASUS (2000) & Positiva \\
\hline
\end{tabular}


Quadro 1 - Indicadores do modelo de avaliação hidroambiental (continuação)

\begin{tabular}{|c|c|c|c|}
\hline $\begin{array}{l}\text { CATEGORIAI } \\
\text { DIMENSÃO }\end{array}$ & INDICADOR & FONTE & $\begin{array}{l}\text { TIPO DE } \\
\text { RELAÇÃO } \\
\text { DO } \\
\text { INDICADOR }\end{array}$ \\
\hline \multirow{5}{*}{$\begin{array}{l}\text { DESEMPENHO DO } \\
\text { SISTEMA QUANTO } \\
\text { AOS INDICADORES } \\
\text { ECONÔMICOS }\end{array}$} & PIB per capita em reais & IBGE (2015) & Positiva \\
\hline & $\begin{array}{l}\text { Receitas de impostos e } \\
\text { transferências de recursos }\end{array}$ & DATASUS (2000) & Positiva \\
\hline & Despesa total com saúde & DATASUS (2000) & Positiva \\
\hline & $\begin{array}{c}\text { Despesa total com saúde } \\
\text { por habitante }\end{array}$ & DATASUS (2000) & Positiva \\
\hline & $\begin{array}{c}\text { Transferência SUS por } \\
\text { habitante }\end{array}$ & DATASUS (2000) & Positiva \\
\hline \multirow{7}{*}{$\begin{array}{l}\text { DESEMPENHO DO } \\
\text { SISTEMA QUANTO AO } \\
\text { ABASTECIMENTO } \\
\text { HUMANO }\end{array}$} & $\begin{array}{l}\text { Densidade demográfica } \\
\left(\mathrm{hab} / \mathrm{km}^{2}\right)\end{array}$ & IBGE (2010) & Positiva \\
\hline & População total & IBGE (2010) & Positiva \\
\hline & Precipitação média anual & $\begin{array}{c}\text { CEPAGRI/UNICA } \\
\text { MP (S/D) }\end{array}$ & Positiva \\
\hline & $\begin{array}{c}\text { Consumo médio per } \\
\text { capita } \\
\end{array}$ & SNIS (2015) & Negativa \\
\hline & $\begin{array}{c}\text { \% abastecimento por rede } \\
\text { geral D }\end{array}$ & SNIS (2015) & Negativa \\
\hline & $\begin{array}{c}\% \text { de abastecimento por } \\
\text { poço nascente }\end{array}$ & SNIS (2016) & Negativa \\
\hline & $\begin{array}{c}\text { \% Outra forma de } \\
\text { abastecimento de água }\end{array}$ & SNIS (2017) & Negativa \\
\hline \multirow{9}{*}{$\begin{array}{l}\text { DESEMPENHO DO } \\
\text { SISTEMA QUANTO ÀS } \\
\text { PRESSÕES DA } \\
\text { IRRIGAÇÃO, } \\
\text { PECUÁRIA, } \\
\text { ABASTECIMENTO } \\
\text { RURAL, } \\
\text { AQUICULTURA E } \\
\text { LAZER }\end{array}$} & $\begin{array}{c}\text { Vazão total outorgada } \\
\text { para captações } \\
\text { superficiais: } \mathrm{m}^{3} / \mathrm{s}\end{array}$ & SIGRH (2016) & Negativa \\
\hline & $\begin{array}{c}\text { Vazão total outorgada } \\
\text { para captações } \\
\text { subterrâneas: } \mathrm{m}^{3} / \mathrm{s}\end{array}$ & SIGRH (2016) & Negativa \\
\hline & $\begin{array}{c}\text { Vazão outorgada para uso } \\
\text { urbano / Volume estimado } \\
\text { para Abastecimento } \\
\text { Urbano: } \%\end{array}$ & SIGRH (2016) & Negativa \\
\hline & Número de bovinos & IBGE (2016) & Negativa \\
\hline & Número de equinos & IBGE (2016) & Negativa \\
\hline & Número de suínos & IBGE (2016) & Negativa \\
\hline & Números de galináceos & IBGE (2016) & Negativa \\
\hline & Número de ovinos & IBGE (2016) & Negativa \\
\hline & Número de caprinos & IBGE (2016) & Negativa \\
\hline
\end{tabular}


Quadro 1 - Indicadores do modelo de avaliação hidroambiental (conclusão)

\begin{tabular}{|c|c|c|c|}
\hline $\begin{array}{l}\text { CATEGORIAI } \\
\text { DIMENSÃO }\end{array}$ & INDICADOR & FONTE & $\begin{array}{l}\text { TIPO DE } \\
\text { RELAÇÃO } \\
\text { DO } \\
\text { INDICADOR }\end{array}$ \\
\hline \multirow{6}{*}{$\begin{array}{c}\text { DESEMPENHO DO } \\
\text { SISTEMA QUANTO A } \\
\text { COLETA DE ESGOTOS }\end{array}$} & $\begin{array}{c}\text { \% rede sanitária via } \\
\text { esgoto }\end{array}$ & DATASUS (2000) & Positiva \\
\hline & $\begin{array}{l}\text { \% rede sanitária via fossa } \\
\text { séptica }\end{array}$ & DATASUS (2000) & Negativa \\
\hline & $\begin{array}{l}\text { \% rede sanitária via fossa } \\
\text { rudimentar }\end{array}$ & DATASUS (2000) & Negativa \\
\hline & \% rede sanitária via vala & DATASUS (2000) & Negativa \\
\hline & $\begin{array}{l}\text { \% rede sanitária via rio ou } \\
\text { lago }\end{array}$ & DATASUS (2000) & Negativa \\
\hline & $\begin{array}{c}\text { \% que não dispõe de } \\
\text { instalação sanitária }\end{array}$ & DATASUS (2000) & Negativa \\
\hline \multirow{4}{*}{$\begin{array}{l}\text { DESEMPENHO DO } \\
\text { SISTEMA QUANTO AO } \\
\text { ATENDIMENTO DE } \\
\text { COLETA DE LIXO }\end{array}$} & $\%$ de lixo coletado & DATASUS (2000) & Positiva \\
\hline & \% lixo queimado & DATASUS (2000) & Negativa \\
\hline & $\%$ lixo enterrado & DATASUS (2000) & Negativa \\
\hline & $\%$ lixo jogado & DATASUS (2000) & Negativa \\
\hline \multirow{5}{*}{$\begin{array}{c}\text { DESEMPENHO DO } \\
\text { SISTEMA QUANTO AO } \\
\text { ESTADO QUALITATIVO } \\
\text { DA ÁGUA }\end{array}$} & Turbidez fora do padrão & SNIS (2015) & Negativa \\
\hline & $\begin{array}{l}\text { Cloro residual fora do } \\
\text { padrão }\end{array}$ & SNIS (2015) & Negativa \\
\hline & Turbidez & SNIS (2015) & Negativa \\
\hline & Coliformes totais & SNIS (2015) & Negativa \\
\hline & $\begin{array}{l}\text { Coliformes totais fora do } \\
\text { padrão }\end{array}$ & SNIS (2015) & Negativa \\
\hline
\end{tabular}

Fonte: Elaborado pelos autores (2018)

O cálculo de cada indicador foi realizado utilizando os parâmetros expostos por Martins e Cândido (2008) para cada localidade, os quais foram adaptados para a determinação da sustentabilidade hidroambiental. Foi realizada a agregação desses indicadores por dimensão através da média aritmética, chegando-se ao ISHM dos municípios que estão sendo avaliados. O ISHM dos municípios com sede totalmente contida na bacia hidrográfica do rio Mogi Guaçu, SP, foi calculado através da média aritmética dos ISHM das categorias/dimensões dos municípios. A Tabela 1 mostra a classificação e representação dos índices (variação 0 e 1): 
Tabela 1 - Classificação e representação dos índices em níveis de sustentabilidade hidroambiental

\begin{tabular}{ccc}
\hline Índice (0 - 1) & Coloração & Performance \\
\hline $0,7501-1,000$ & & Ideal \\
$0,5001-0,7500$ & & Aceitável \\
$0,2501-0,5000$ & & Alerta \\
$0,0000-0,2500$ & & Crítica \\
\hline
\end{tabular}

Fonte: Adaptado para o estudo conforme Martins e Cândido (2008)

Os índices de sustentabilidade com valores entre 0,0000 e 0,2500 são caracterizados pela performance que revela um estado crítico de sustentabilidade; os índices com valores entre 0,2501 e 0,5000 foram representados pela performance que revela um estado ou situação de alerta; os índices com valores entre 0,5001 e 0,7000 foram representados pela performance que revela um estado ou situação aceitável; e por último, os índices com valores entre 0,7001 e 1,0000 foram representados pela performance que revela estado ou situação ideal de sustentabilidade (MARTINS e CÂNDIDO, 2008).

A bacia hidrográfica utilizada no estudo é a bacia hidrográfica do Rio Mogi Guaçu (CBH-MOGI) da qual fazem parte os municípios com sede urbana na área de drenagem: Aguaí, Águas da Prata, Águas de Lindóia, Américo Brasiliense, Araras, Barrinha, Conchal, Descalvado, Dumont, Engenheiro Coelho, Espírito Santo do Pinhal, Estiva Gerbi, Guariba, Guatapará, Itapira, Jaboticabal, Leme, Lindóia, Luís Antônio, Mogi Guaçu, Mogi Mirim, Motuca, Pirassununga, Pitangueiras, Pontal, Porto Ferreira, Pradópolis, Rincão, Santa Cruz da Conceição, Santa Cruz das Palmeiras, Santa Lúcia, Santa Rita do Passa Quatro, Santo Antônio do Jardim, São João da Boa Vista, Serra Negra, Sertãozinho, Socorro e Taquaral (SIGRH, 2015).

Neste trabalho foram analisados todos os municípios com sede totalmente contida na bacia hidrográfica do Rio Mogi Guaçu, SP (Águas da Prata, Engenheiro Coelho, Luís Antônio, Mogi Mirim, Pitangueiras, São João da Boa Vista, Serra Negra, Sertãozinho, Socorro e Taquaral) conforme definido pelo Relatório de Situação dos Recursos Hídricos (SIGRH, 2015).

A bacia hidrográfica do rio Mogi Guaçu, em escala federal, pertence à Região Hidrográfica do Paraná que é constituída pela bacia hidrográfica do rio Paraná situada no território nacional. Em escala estadual, pertence ao Estado de São Paulo e constitui a Unidade de Gerenciamento de Recursos Hídricos 09 - UGRHI 09 localizada a noroeste do 
Estado (SIGRH, 2015). A Figura 1 ilustra a localização da Bacia Mogi Guaçu no Estado de São Paulo.

Figura 3 - Localização da Bacia Mogi Guaçu no Estado de São Paulo

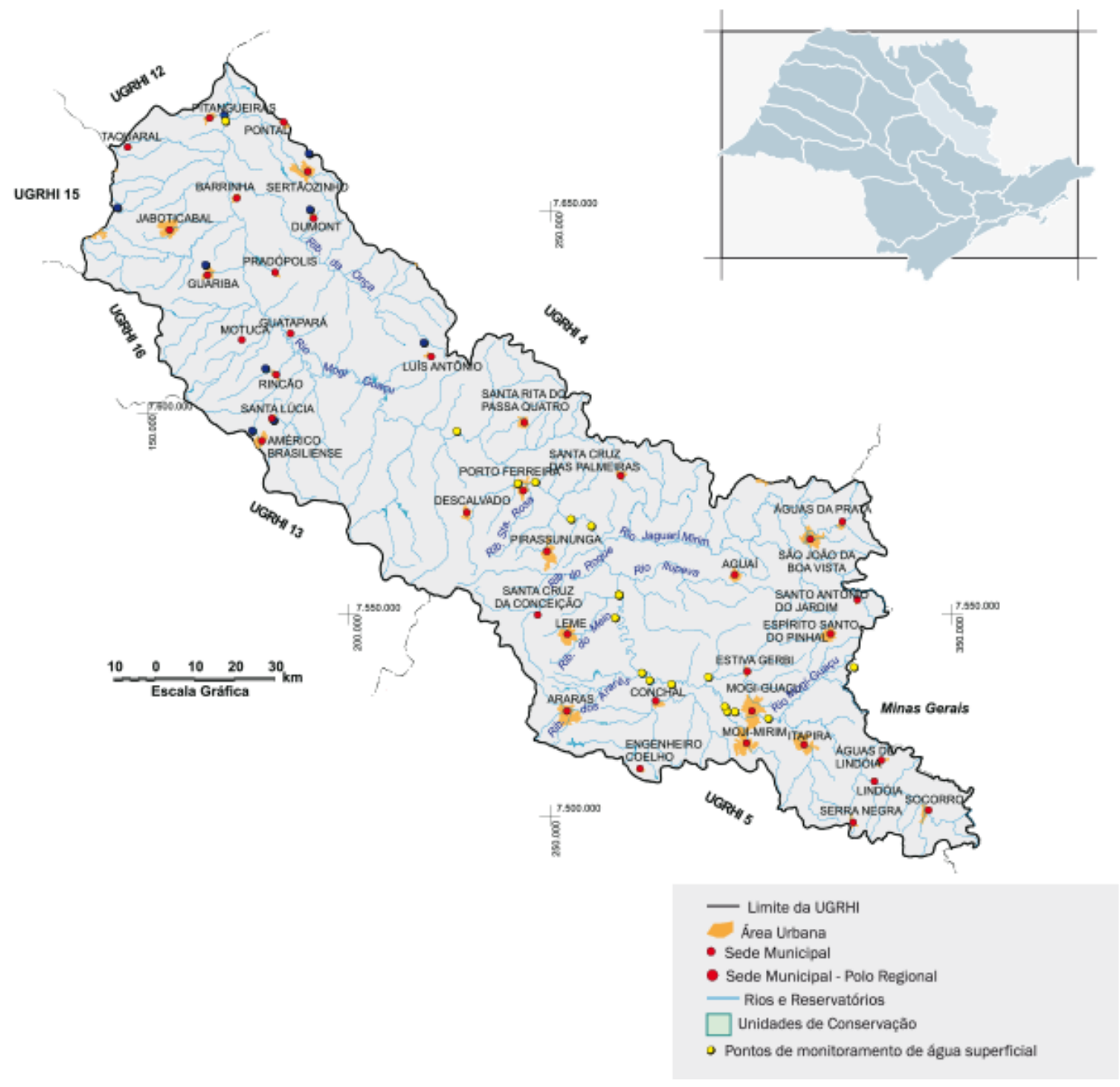

Fonte: SIGRH (2015)

A seguir estão evidenciados os resultados relacionados à caracterização dos indicadores de cada uma das dimensões analisadas e consideradas no estudo.

\section{RESULTADOS E DISCUSSÃO}

A partir dos indicadores relacionados ao desempenho do sistema quanto ao desenvolvimento humano de cada município (Quadro 1) e após as análises positivas e 
negativas de cada um deles foi possível chegar aos resultados que estão evidenciados nas Tabelas 2, 3, 4 e 5.

Tabela 2 - Desempenho dos Municípios quanto ao Desenvolvimento Humano e Indicadores Econômicos

\begin{tabular}{|c|c|c|c|c|}
\hline \multirow{2}{*}{ Municípios } & \multicolumn{2}{|c|}{$\begin{array}{l}\text { Desenvolvimento } \\
\text { Humano }\end{array}$} & \multicolumn{2}{|c|}{ Indicadores Econômicos } \\
\hline & $\begin{array}{l}\text { Média do } \\
\text { Município }\end{array}$ & Performance & $\begin{array}{l}\text { Média do } \\
\text { Município }\end{array}$ & Performance \\
\hline Águas da Prata & 0,6425 & Aceitável & 0,0575 & Crítica \\
\hline Engenheiro Coelho & 0,2453 & Crítica & 0,1370 & Crítica \\
\hline Luís Antônio & 0,3929 & Alerta & 0,5153 & Aceitável \\
\hline Mogi Mirim & 0,6602 & Aceitável & 0,6832 & Aceitável \\
\hline Pitangueiras & 0,4044 & Alerta & 0,1760 & Crítica \\
\hline São João da Boa Vista & 0,6529 & Aceitável & 0,5247 & Aceitável \\
\hline Serra Negra & 0,6140 & Aceitável & 0,1165 & Crítica \\
\hline Sertãozinho & 0,6778 & Aceitável & 0,5688 & Aceitável \\
\hline Socorro & 0,4127 & Alerta & 0,2181 & Crítica \\
\hline Taquaral & 0,1190 & Crítica & 0,2331 & Crítica \\
\hline $\begin{array}{l}\text { Média dos } \\
\text { municípios }\end{array}$ & 0,4822 & Alerta & 0,3230 & Alerta \\
\hline
\end{tabular}

Fonte: Resultados da pesquisa. Elaborado pelos autores

$\mathrm{Na}$ Tabela 2 observa-se, quanto ao Desenvolvimento Humano, que dos dez municípios estudados, cinco (50\%) apresentaram uma situação de alerta ou crítica, a saber: Engenheiro Coelho (0,2453), Luís Antônio (0,3929), Pitangueiras (0,4044), Socorro $(0,4127)$ e Taquaral $(0,1190)$. Os demais $(50 \%)$ apresentaram performance aceitável: Águas da Prata (0,6425), Mogi Mirim (0,6602), São João da Boa Vista $(0,6529)$, Serra Negra $(0,6140)$ e Sertãozinho $(0,6778)$. Verifica-se que dentre os dez municípios, nenhum deles apresentou situação ideal. Nota-se que a média geral dos municípios com sede totalmente contida na bacia, é de alerta $(0,4822)$ na ótica do desenvolvimento humano. Fica evidente a necessidade de melhorar essa situação em busca da situação ideal de modo que possa contribuir satisfatoriamente para esse contexto geográfico. Entre os indicadores que precisam ser melhorados destacam-se: a quantidade de estabelecimentos de saúde (SUS) e a população coberta pelo Programa de Saúde da Família (PSF).

Para os Indicadores Econômicos, seis municípios (60\%) apresentaram uma situação crítica: Águas da Prata (0,0575), Engenheiro Coelho $(0,1370)$, Pitangueiras $(0,1760)$, Serra Negra $(0,1165)$, Socorro $(0,2181)$ e Taquaral $(0,2331)$. Outros quatro municípios $(40 \%)$ 
apresentaram performance aceitável: Luís Antônio (0,5153), Mogi Mirim (0,6832), São João da Boa Vista $(0,5247)$ e Sertãozinho $(0,5688)$. Nota-se que a média geral dos municípios com sede totalmente contida na bacia, é de alerta $(0,3230)$ levando-se em conta os Indicadores Econômicos. Entre os indicadores que precisam ser melhorados destacam-se: PIB (Produto Interno Bruto) per capita, despesa total com saúde por habitante e transferência SUS por habitante.

As médias dos municípios estudados, por apresentarem performance alerta para Desenvolvimento Humano e Indicadores Econômicos, são fortes entraves da bacia hidrográficas Mogi Guaçu, posto que se considera a água como um vetor que contribui para a melhoria da qualidade hidroambiental.

Em relação ao desempenho dos municípios quanto aos indicadores de Saneamento (Tabelas 3 e 4), quanto ao abastecimento humano, três municípios apresentam performance alerta: Luís Antônio (0,3691), Pitangueiras $(0,2600)$ e Serra Negra $(0,4798)$. Apenas o município de Águas da Prata $(0,4204)$ apresentou performance crítica, e os demais municípios (60\%) apresentam performance aceitável. Cabe ressaltar que embora a média dos municípios, para abastecimento humano, tenha alcançado performance aceitável $(0,5455)$, são necessários esforços na tentativa de melhorar este desempenho, já que entre as categorias/dimensões de Saneamento (Tabelas 3 e 4), foi a que apresentou menor performance.

Para coleta de esgoto, apenas o município de Socorro $(0,2966)$ apresentou performance alerta. Os demais (90\%) apresentaram performance aceitável ou ideal. Destaque para os municípios de Luís Antônio $(0,7762)$, São João da Boa Vista $(0,7575)$, Sertãozinho $(0,7610)$ e Taquaral $(0,8077)$ que apresentaram uma performance ideal.

Na coleta de lixo, dois municípios apresentaram performance alerta: Águas da Prata $(0,2959)$ e Serra Negra $(0,4887)$, já os demais $(80 \%)$ apresentaram performance aceitável. 
Tabela 3 - Desempenho dos Municípios quanto aos indicadores de Abastecimento Humano e Estado Qualitativo da Água

\begin{tabular}{|c|c|c|c|c|}
\hline \multirow{2}{*}{ Municípios } & \multicolumn{2}{|c|}{ Abastecimento Humano } & \multicolumn{2}{|c|}{$\begin{array}{c}\text { Estado Qualitativo da } \\
\text { Água }\end{array}$} \\
\hline & $\begin{array}{l}\text { Média do } \\
\text { Município }\end{array}$ & Performance & $\begin{array}{l}\text { Média do } \\
\text { Município }\end{array}$ & Performance \\
\hline Águas da Prata & 0,4204 & Crítica & 0,5420 & Aceitável \\
\hline Engenheiro Coelho & 0,5737 & Aceitável & 0,9981 & Ideal \\
\hline Luís Antônio & 0,3691 & Alerta & 0,8000 & Ideal \\
\hline Mogi Mirim & 0,5413 & Aceitável & 1,0000 & Ideal \\
\hline Pitangueiras & 0,2600 & Alerta & 1,0000 & Ideal \\
\hline São João da Boa Vista & 0,6259 & Aceitável & 0,8845 & Ideal \\
\hline Serra Negra & 0,4798 & Alerta & 0,9145 & Ideal \\
\hline Sertãozinho & 0,7412 & Aceitável & 0,8000 & Ideal \\
\hline Socorro & 0,5470 & Aceitável & 0,6829 & Aceitável \\
\hline Taquaral & 0,8961 & Ideal & -- & -- \\
\hline $\begin{array}{l}\text { Média dos } \\
\text { municípios }\end{array}$ & 0,5455 & Aceitável & 0,8469 & Ideal \\
\hline
\end{tabular}

Fonte: Resultados da pesquisa. Elaborado pelos autores

Tabela 4 - Desempenho dos Municípios quanto aos indicadores de Coleta de Esgotos e Coleta de Lixo

\begin{tabular}{lcccc}
\hline \multirow{2}{*}{ Municípios } & \multicolumn{2}{c}{ Coleta de Esgotos } & \multicolumn{2}{c}{ Coleta de Lixo } \\
\cline { 2 - 5 } & $\begin{array}{c}\text { Média do } \\
\text { Município }\end{array}$ & Performance & $\begin{array}{c}\text { Média do } \\
\text { Município }\end{array}$ & Performance \\
\hline Águas da Prata & 0,6715 & Aceitável & 0,2959 & Alerta \\
Engenheiro Coelho & 0,6687 & Aceitável & 0,6928 & Aceitável \\
Luís Antônio & 0,7762 & Ideal & 0,6062 & Aceitável \\
Mogi Mirim & 0,7218 & Aceitável & 0,7010 & Aceitável \\
Pitangueiras & 0,7805 & Aceitável & 0,7337 & Aceitável \\
São João da Boa & 0,7575 & Ideal & 0,6912 & Aceitável \\
Vista & 0,5166 & Aceitável & 0,4887 & Alerta \\
Serra Negra & 0,7610 & Ideal & 0,6667 & Aceitável \\
Sertãozinho & 0,2966 & Alerta & 0,5476 & Aceitável \\
Socorro & 0,8077 & Ideal & 0,6023 & Aceitável \\
Taquaral & $\mathbf{0 , 6 7 5 8}$ & Aceitável & $\mathbf{0 , 6 0 2 6}$ & Aceitável \\
\hline Média dos & & &
\end{tabular}

Fonte: Resultados da pesquisa. Elaborado pelos autores

Quanto ao estado qualitativo da água todos os municípios estudados, com exceção de Taquaral que não dispunha de dados, apresentaram performance aceitável ou ideal. No geral, sete municípios (Engenheiro Coelho, Luís Antônio, Mogi Mirim, Pitangueiras, São 
João da Boa Vista, Serra Negra e Sertãozinho) apresentaram desempenhos satisfatórios, visto que, a performance geral ficou ideal (acima de 0,8000).

Nota-se que a média geral dos municípios para abastecimento humano $(0,5455)$, coleta de esgoto $(0,6758)$ e coleta de lixo $(0,6026)$ apresentaram performance aceitável; e para o estado qualitativo da água $(0,8469)$ performance ideal. Sendo assim, quanto aos indicadores de Saneamento, a média dos municípios são satisfatórias para bacia hidrográfica do rio Mogi Guaçu.

Finalmente, quanto a categoria/dimensão desempenho dos municípios quanto às Pressões da Irrigação, Pecuária, Abastecimento Rural, Aquicultura e Lazer (Tabela 5), estes apresentaram média 0,7641 , ou seja, performance ideal, sendo que $90 \%$ dos municípios tem performance ideal ou aceitável, com exceção de Mogi Mirim $(0,3690)$ cuja performance é alerta.

\begin{tabular}{lcc}
$\begin{array}{c}\text { Tabela } 5 \\
\text { - Desempenho dos Municípios quanto às Pressões da } \\
\text { Irrigação, Pecuária, Abastecimento Rural, Aquicultura }\end{array}$ \\
\hline Municípios & $\begin{array}{c}\text { Média do } \\
\text { Município }\end{array}$ & Performance \\
\hline Águas da Prata & 0,8433 & Ideal \\
Engenheiro Coelho & 0,9030 & Ideal \\
Luís Antônio & 0,7670 & Ideal \\
Mogi Mirim & 0,3690 & Alerta \\
Pitangueiras & 0,8821 & Ideal \\
São João da Boa Vista & 0,7916 & Ideal \\
Serra Negra & 0,7559 & Ideal \\
Sertãozinho & 0,6589 & Aceitável \\
Socorro & 0,6812 & Aceitável \\
Taquaral & 0,9888 & Ideal \\
\hline Média dos municípios & $\mathbf{0 , 7 6 4 1}$ & Ideal \\
\hline \multicolumn{2}{c}{ Fonte: Resultados da pesquisa. Elaborado pelos autores }
\end{tabular}

Segundo dados analisados, Mogi Mirim está sujeito a fortes pressões relacionadas a pecuária (número de equinos, suínos, galináceos, ovinos e caprinos) sendo necessárias políticas públicas que visem mitigar tais pressões sobre os recursos hídricos. 


\section{CONCLUSÕES}

Dos indicadores analisados, nota-se que Desenvolvimento Humano e os Indicadores Econômicos apresentam situações mais preocupantes na bacia por terem performance de alerta. Destaca-se para o Desenvolvimento Humano a necessidade de ações relacionados a saúde da população; e para os Indicadores Econômicos é preciso investimentos do setor público e privado a fim de gerar mais emprego e renda, aumentando desta forma o PIB (Produto Interno Bruto) per capita nos municípios; além também de promover ações de saúde preventiva para diminuir as despesas com saúde por habitante.

Em relação ao desempenho dos municípios, quanto aos Indicadores de Saneamento, o abastecimento humano foi o mais preocupante, já que $40 \%$ dos municípios apresentaram performance alerta ou crítica. Foi por esta razão que esta categoria/dimensão apresentou menor performance entre todas as médias municipais apuradas entre os Indicadores de Saneamento. São necessárias políticas públicas e maiores investimentos dos gestores municipais e Comitê de Bacia neste indicador, priorizando o abastecimento de domicílio por água proveniente de uma rede geral de distribuição.

Embora às Pressões da Irrigação, Pecuária, Abastecimento Rural, Aquicultura e Lazer apresentem performance ideal ou aceitável para 90\% dos municípios (exceção de Mogi Mirim cuja performance é alerta), tais pressões precisam ser monitoradas periodicamente pela afinidade da bacia no setor agropecuário.

Por fim, neste estudo foi possível notar a importância desta metodologia para análise integrada dos indicadores de sustentabilidade hidroambiental da bacia hidrográfica. As categorias/dimensões (desenvolvimento humano; econômico; abastecimento humano; pressões da irrigação, pecuária, abastecimento rural, aquicultura e lazer; coleta de esgoto; atendimento de coleta de lixo; e estado qualitativo da água) revelaram situações precárias em alguns municípios e que podem ser ajustadas pelas políticas públicas Municipais, Estaduais e Federais. Além disso, tais dados podem servir de parâmetros ações e diretrizes nos gestores do Comitê de Bacia Hidrográfica do Mogi Guaçu.

\section{AGRADECIMENTOS}

A Fundação CAPES (Coordenação de Aperfeiçoamento de Pessoal de Nível 
Superior), MEC (Ministério da Educação), PROSUP (Programa de Suporte à PósGraduação de Instituições de Ensino Particulares).

\section{REFERÊNCIAS}

BRASIL. LEI No 9.433, DE 8 DE JANEIRO DE 1997. Institui a Política Nacional de Recursos Hídricos, cria o Sistema Nacional de Gerenciamento de Recursos Hídricos, regulamenta o inciso XIX do art. 21 da Constituição Federal, e altera o art. $1^{\circ}$ da Lei ${ }^{\circ}$ 8.001, de 13 de março de 1990, que modificou a Lei $n^{0} 7.990$, de 28 de dezembro de 1989. Diário Oficial [da] República Federativa do Brasil. Brasília, 1997.

CARVALHO, J. R. M, CÂNDIDO G. A., CURI, W. F, ARAÚJO, E. K. M. Análise da sustentabilidade hidroambiental de municípios da região da sub-bacia do Alto Piranhas, PB. HOLOS, v. 6, 2015. Disponível em: < http://www2.ifrn.edu.br/ojs/index.php/HOLOS/article/view/2093/1210> . Acesso em: 10 janeiro 2018.

DOWBOR, Ladislau. Economic democracy: meeting some management challenges: changing scenarios in Brazil. Journal of the European Academy of Science and Arts, v. 8, n. 2, jul. 2013.

GARCIA, E. N. A.; MORENO, D.A.A.C.; FERNANDES, A.L.V. A importância da preservação e conservação das águas superficiais e subterrâneas: um panorama sobre a escassez da água no Brasil. FÓRUM AMBIENTAL DA ALTA PAULISTA, 11., 2015. Anais... v. 11. n. 6, 2015, p. 235249.

GUIMARÃES, L. T. Proposta de um sistema de indicadores de desenvolvimento sustentável para bacias hidrográficas. 2008. 237 f. Tese (Doutorado em Ciências em Planejamento Energético) - Programa de Pós-graduação de Engenharia da Universidade Federal do Rio de Janeiro, Rio de Janeiro, RJ, 2008.

MACHADO, P. A. L. Direito ambiental brasileiro. 23. ed. São Paulo: Malheiros, 2015.

MAGALHÃES JÚNIOR, A. P. Indicadores ambientais e recursos hídricos: realidade e perspectivas para o Brasil a partir da experiência francesa. 2. ed. Rio de Janeiro, RJ: Bertrand Brasil, 2010.

MARTINS, M. F., CÂNDIDO, G. A. Índice de desenvolvimento sustentável para municípios (IDSM): metodologia para análise e cálculo do IDSM e classificação dos níveis de sustentabilidade - uma aplicação no Estado da Paraíba. João Pessoa, PB: SEBRAE-PB, 2008.

SIGRH. Sistema Integrado de Gerenciamento dos Recursos Hídricos. Relatório de Situação dos Recursos Hídricos 2015. Comitê da bacia hidrográfica do rio Mogi Guaçu (UGRHI $09 \mathrm{CBH}$ MOGI). Disponível em www.pha.poli.usp.br/LeArq.aspx?id arq=5059. Acesso: 11 de janeiro de 2018.

SEPÚLVEDA, S. Desenvolvimento microrregional sustentável: métodos para planejamento local. Brasília: IICA, 2005. 
SOUZA, L. C. L; MATOS, I. M. A; PETER, M. G. A; MACHADO, M. V. V; NASCIMENTO, C. P. S. Índice de desenvolvimento sustentável para municípios (IDSM): um estudo sobre o nível de sustentabilidade das capitais brasileiras. ENGEMA, 15., Anais.... 2014 . Disponível em: http://www.engema.org.br/XVIENGEMA/431.pdf. Acesso: 17 de janeiro de 2018.

VIEIRA, P. M. S.; STUDART, T. M. C. Proposta metodológica para o desenvolvimento de um índice de sustentabilidade hidroambiental de áreas serranas no semiárido brasileiro - estudo de caso: Maciço de Baturité, Ceará. Revista Brasileira de Recursos Hídricos, v.14, n. 4, p. 125136, out./dez, 2009.

WAQUIL, P. D.; SCHNEIDER, S.; FILIPPI, E. E.; CONTERATO, M. A.; SPECHT, S. Para medir o desenvolvimento territorial rural: validação de uma proposta metodológica. In: CONGRESSO DA SOCIEDADE BRASILEIRA DE ECONOMIA, ADMINISTRAÇÃO E SOCIOLOGIA RURAL, 44. 2007. Anais... Londrina, PR. Anais. Londrina/PR: Universidade Estadual de Londrina, PR, 2007. 